\title{
Modeling Precipitate Evolution in Zirconium Alloys during Irradiation
}

DOI:

10.1016/j.jnucmat.2016.04.036

\section{Document Version}

Accepted author manuscript

Link to publication record in Manchester Research Explorer

\section{Citation for published version (APA):}

Robson, J. (2016). Modeling Precipitate Evolution in Zirconium Alloys during Irradiation. Journal of Nuclear Materials, 476, 123-131. https://doi.org/10.1016/j.jnucmat.2016.04.036

\section{Published in:}

Journal of Nuclear Materials

\section{Citing this paper}

Please note that where the full-text provided on Manchester Research Explorer is the Author Accepted Manuscript or Proof version this may differ from the final Published version. If citing, it is advised that you check and use the publisher's definitive version.

\section{General rights}

Copyright and moral rights for the publications made accessible in the Research Explorer are retained by the authors and/or other copyright owners and it is a condition of accessing publications that users recognise and abide by the legal requirements associated with these rights.

\section{Takedown policy}

If you believe that this document breaches copyright please refer to the University of Manchester's Takedown Procedures [http://man.ac.uk/04Y6Bo] or contact uml.scholarlycommunications@manchester.ac.uk providing relevant details, so we can investigate your claim.

\section{OPEN ACCESS}




\title{
Modeling Precipitate Evolution in Zirconium Alloys during Irradiation
}

\author{
J. D. Robson ${ }^{\mathrm{a}, *}$ \\ ${ }^{a}$ School of Materials, University of Manchester, MSS Tower, Manchester, \\ M13 9PL, UK
}

\begin{abstract}
The second phase precipitates (SPPs) in zirconium alloys are critical in controlling their performance. During service, SPPs are subject to both thermal and irradiation effects that influence volume fraction, number, and size. In this paper, a model has been developed to capture the combined effect of thermal and irradiation exposure on the $\mathrm{Zr}(\mathrm{Fe}, \mathrm{Cr})_{2}$ precipitates in Zircaloy. The model includes irradiation induced precipitate destabilisation integrated into a classical size class model for nucleation, growth and coarsening. The model has been applied to predict the effect of temperature and irradiation on SPP evolution. Increasing irradiation displacement rate is predicted to strongly enhance the loss of particles that arises from coarsening alone. The effect of temperature is complex due to competition between coarsening and irradiation damage. As temperature increases, coarsening is predicted to become increasingly important compared to irradiation induced dissolution and may increase resistance to irradiation induced dissolution by increasing particle size.
\end{abstract}

Key words:

\section{INTRODUCTION}

Second phase precipitates (SPPs) are known to have a strong influence on the performance of zirconium alloys used for in-reactor components. Corrosion behaviour depends critically on the volume fraction, size, and distribution of the SPPs, which must be optimised to obtain the best performance. For example, in Zircaloy-4 it has been demonstrated that the optimum precipitate size distribution must be in a range where the particles are sufficiently fine

* Corresponding author.

Email address: joseph.robson@manchester.ac.uk (J. D. Robson). 
to give good nodular corrosion resistance whilst remaining above a critical minimum size that is required for good uniform corrosion resistance $[1,2]$. SPPs and the solute from which they are formed also have a key role in determining other properties such as strength and susceptibility to irradiation growth $[3-5]$.

SPPs are formed during the initial processing of the zirconium alloys. The standard processing sequence involves quenching from the high temperature $\beta$ phase field followed by thermomechanical processing and annealing with zirconium in the $\alpha$ phase field. It is during processing in the $\alpha$-range that the supersaturated alloying elements (e.g. Fe and $\mathrm{Cr}$ in Zircaloy-4) precipitate as SPPs. In Zircaloy-4, the dominant precipitate phases are intermetallics of composition $\mathrm{Zr}(\mathrm{Fe}, \mathrm{Cr})_{2}$ and can take either hexagonal or face centred cubic allotropic forms [2].

Since the precipitates have a strong influence on alloy performance, their stability during in-reactor service is a critical concern. Any changes in the nature and distribution of the SPPs in service will lead to a commensurate change in corrosion performance, potentially leading to accelerated in-reactor corrosion. Furthermore, dissolution of the precipitates will increase the concentration of alloying elements in solution in the matrix and this can promote the formation of $\langle\mathrm{c}\rangle$-component dislocation loops, which has been correlated with an increase in irradiation growth $[3-5]$.

In the reactor, SPPs are subject to both thermal and irradiation exposure which leads to evolution of the particles during service. Thermal exposure alone will lead to coarsening of the SPP distribution driven by a reduction in the total surface area of the precipitates. Irradiation adds considerable complexity to the particle evolution behaviour. Not only can irradiation enhance the kinetic processes that occur during thermal exposure alone, but irradiation also introduces entirely new phenomena. Principal amongst these is a change in composition and sometimes crystallinity of the SPPs, followed by their dissolution. For example, in a range of zirconium alloys it has been observed that SPPs become denuded in certain elements (e.g. Fe) after irradiation exposure, and the thickness of the denuded layer grows linearly with time (for a constant dose rate). This can also be accompanied by amorphization of the crystalline SPP phase in some cases $[3,4,6-8]$.

The exact mechanism by which irradiation alters the composition and structure of SPPs remains under debate and a number of models have been developed to predict this process based on different proposed physical processes $[3,6,8,9]$.

Critically, from the perspective of maintaining a desirable SPP distribution, irradiation damage and composition change will destabilise the SPPs, and 
eventually can lead to their complete dissolution [3,6]. This is a multistep process since in many commercial zirconium alloys (e.g. Zircaloys, Zirlo) the dominant SPPs contain both faster (e.g. Fe) and slower (e.g. Cr, Nb) diffusing species [10]. The first stage in irradiation damage of SPPs often involves loss of the faster diffusing species (e.g. Fe) from the SPPs, but full dissolution also requires diffusion of the slow diffusing species away from the precipitate, so takes longer. It remains unclear how much overlap there is between the various kinetic processes that occur during SPP exposure to irradiation. However, the size of irradiated SPPs reported in the literature suggest that for the dominant cubic $\mathrm{Zr}(\mathrm{Cr}, \mathrm{Fe})_{2}$ SPPs in Zircaloy-4 in the intermediate temperature range $(T=520-580 \mathrm{~K})$ composition change and amorphization usually occurs prior to a large size change due to dissolution [3,7-9]. Temperature plays a key role in determining the observed behaviour since the rates of both amorphization and dissolution are expected to be temperature dependent, but with different sensitivities. This means that the amount of overlap between these processes will change with temperature. This is discussed in detail elsewhere [3].

The microstructure that results from SPP dissolution is likely to make the alloy more susceptible to corrosion and irradiation growth. It is thus of key importance to understand (and ideally simulate) the SPP evolution during in reactor service so that exposure conditions that lead to an unacceptable change in SPP distribution can be identified. A simulation tool to predict SPP evolution in service would also be of great value in helping to design irradiation resistant microstructures.

In the present work, a simple coupled physical model has been developed that provides a prediction of SPP evolution under conditions where particle amorphization and dissolution occurs. The uncertainty in some of the mechanisms that determine the effect of irradiation on SPPs mean that the model is not expected to be quantitatively accurate. However, it is a useful tool in exploring the consequences of irradiation effects on SPPs in-reactor and qualitatively predict the influence of key variables (temperature and irradiation displacement rate) on SPP evolution.

\section{THE MODEL}

Precipitate evolution under irradiation is a highly complex problem and is not yet fully understood. However, sufficient is known about the key phenomena to capture them in a predictive model that is expected to give qualitatively reasonable, if not quantitatively accurate, predictions.

The approach taken was to couple a model that describes irradiation induced SPP amorphization and destabilization with a kinetic model proven for predic- 
tion of SPP evolution in the absence of irradiation. This model has then been used to explore the SPP evolution with and without irradiation to identify the interaction between irradiation and thermal effects, and identify areas for further study. The model has been applied to Zircaloy-4, since the amoprhization process has been extensively studied in this alloy. In addition, a validated kinetic model has been developed previously for SPPs in Zircaloys can provide many of the input parameters needed in the present work [11]. Only the dominant SPP phase present under the conditions of interest is considered, which is face-centred cubic $\operatorname{Zr}(\mathrm{Cr}, \mathrm{Fe})_{2}[2]$.

It has been demonstrated that irradiation damage of the particles proceeds from particle surface to centre. It has been shown that the kinetics of this process are linear with time for a given dose rate. A simple model has been developed by Motta et al. [8] that captures this linear time dependence, assuming the composition change and destabilization of SPPs is a process that is governed by ballistic effects. This model is summarised later, and full details are provided elsewhere [8].

Composition change and any amorphization that may occur will increase the free energy of the SPPs. Since the change proceeds progressively from the particle surface to edge, but at the same rate for all particles (according to the model of Motta et al. [8]) smaller particles will become fully destabilised before larger particles.

As SPPs dissolve, the solute supersaturation in the matrix will increase, and with it so will the driving force for nucleation of new SPPs. Indeed, it has been shown experimentally that some new SPPs can often be detected after irradiation exposure. However, this reprecipitation problem is highly complex and remains poorly understood. For example, in many cases it appears the reprecipitated SPPs are of a different phase to the original SPP phase [3,4], and may even be of a phase that only occurs under irradiation. Secondly, the reprecipitation process is heterogeneous, and is likely to occur on defects introduced by irradiation, as well as pre-existing favourable nucleation sites (e.g. grain boundaries). It is also not clear how much of the reprecipitation observed experimentally occurs during irradiation, and how much takes place after exposure (e.g. during post-irradiation cooling).

For these reasons, the reprecipitation problem is not considered in the present model; i.e. during irradiation, new particles are not allowed to nucleate. The model therefore captures irradiation and coarsening induced SPP dissolution, and the increase in matrix solute concentration, but re-nucleation is not considered. The consequences of this assumption are explored in detail later. 


\subsection{Irradiation Induced SPP Destabilization Model}

The model used in the present work to predict the effect of irradiation on SPPs is that developed by Motta et al. [8], applied by them to predict amorphization. This model assumes that a departure from the stable composition is a ballistically controlled process that proceeds from the outer to the inner of the particle. This is assumed to spontaneously cause amorphization once the free energy increase in the crystalline state due to the composition change exceeds the free energy change for amorphization. The physical basis of this model and the amorphization step are not critical in the present work; what is important is that this model predicts that the depth of destabilised layer increases linearly with time, and this is in good agreement with experimental observations.

The model gives the depth of amorphization as a function of time [8]:

$$
X_{\text {irrad }}=\frac{1}{6} \mu \phi \sigma_{d} t\left[\frac{3\left(C_{0}-C_{a}\right)}{2 \delta_{C}}-1\right]
$$

where $\mu$ is the average distance travelled by a sputtered atom (taken as $5 \mathrm{~nm}[8]), \phi \sigma_{d}$ is the displacement rate, $C_{0}$ is the concentration of iron in the original (crystalline) SPP, $C_{a}$ is the final concentration of iron measured in amorphous $\operatorname{SPP}(=0.1$ following [8]) and $\delta C$ the composition change required for destabilization of the crystalline SPP phase (taken as 0.03 following [8]). These values have been shown to reproduce the amorphization rates measured experimentally in Zircaloy-4 for temperatures between 520 and $580 \mathrm{~K}$ and doses between 1 and $10 \mathrm{dpa}$ [8]. Note that the displacement rates used in the experiments to validate the model $\left(5 \times 10^{-7} \mathrm{dpas}^{-1}\right)$ are approximately five times greater than that expected in service for a commercial reactor $\left(\approx 1 \times 10^{-7} \mathrm{dpa} \mathrm{s}^{-1}\right)$ [12], and although qualitatively it has been shown that the same phenomena occur at the lower displacement rates, the quantitative predictions are unproven in this regime.

As discussed, the composition change induced by irradiation will destabilise the amorphous SPPs, leading to their dissolution. However, dissolution also requires diffusion of $\mathrm{Cr}$ away from the destabilised region. In the present work, it is assumed that the kinetic processes of irradiation controlled destabilisation and diffusion controlled dissolution are sufficiently separated that they can be treated as sequential and not simultaneous processes. In other words, it is assumed that accelerated dissolution of SPPs only occurs once they have become fully destabilised. This assumption can be justified based on experimental observation in the temperature range $520-580 \mathrm{~K}$; it is seen that particles can become fully amorphous without a large change in their size $[3,8]$. 
It is further assumed that the conventional coarsening process can occur during irradiation; that is, there remain some large particles that are not amorphized and can continue to grow as solute is released into the matrix by dissolution of smaller particles. Under irradiation, the dissolution of small particles is accelerated, which will in turn accelerate the coarsening process. This is handled automatically by the kinetic model framework, and the consequences are discussed in detail later.

Once a particle becomes fully destabilised ( $X_{\text {irrad }}$ reaches the SPP radius), its free energy is accordingly increased in the model. This has the effect of destabilising the particle, leading to its dissolution. Small particles will become destabilised before large particles and will start dissolving first. This will accelerate the normal coarsening process, which is predicted naturally by the kinetic model.

In the present model, it is assumed that once a particle has undergone irradiation induced composition change, it is no longer stable regardless of its size and it will dissolve. This is imposed in the model by setting the critical radius for stability of a destabilised particle as larger than the maximum particle size. Dissolution then occurs naturally within the kinetic model framework (discussed below).

\subsection{Kinetic Model}

The equations for nucleation and growth are coupled using Kampmann and Wagner Numerical (KWN) model, which has been described in detail elsewhere $[13,14]$. In this work, the nucleation and growth model is run concomitantly with the amorphization model. The essential features of the computational implementation of the model are summarized below:

(1) The continuous time evolution of the particle distribution and matrix solute level is considered in terms of discreet time steps.

(2) The continuous particle size distribution (PSD) is discretized into a large number of size classes.

(3) The complex chemistry of Zircaloy-4 is simplified to a pseudo-binary system, with an effective diffusivity used that accounts for all diffusing species, following Massih et al. [11].

(4) The number of new particles in each time step is calculated using classical nucleation theory. Re-nucleation of SPPs during irradiation is not permitted.

(5) The exchange of particles between size classes is calculated assuming solute diffusion is the rate limiting process and a spherical growth morphology. 
Table 1

\begin{tabular}{|cc|}
\hline Parameter & Value \\
\hline Molar volume & $9 \times 10^{-6} \mathrm{~m}^{3} \mathrm{~mol}^{-1}$ \\
Solute concentration in particle & $54 \mathrm{at} . \%$ \\
Effective nucleation site density & $21.5 \times 10^{3} \mu \mathrm{m}^{-3}$ \\
Effective interfacial energy & $0.25 \mathrm{Jm}^{-2}$ \\
Effective activation energy for diffusion & $132.4 \mathrm{~kJ} \mathrm{~mol}^{-1}$ \\
Effective pre-factor for diffusion & $1.473 \times 10^{-6} \mathrm{~m}^{2} \mathrm{~s}^{-1}$ \\
\hline
\end{tabular}

Model input parameters taken from [11]

(6) The Gibbs Thomson relationship [15] is used to calculate the modified interfacial compositions for each size class and at each time step.

(7) Once the amorphization depth exceeds the radius of a given size class all particles in that class are assumed to be amorphized and their dissolution is enforced.

(8) The change in matrix solute level due to precipitate formation or dissolution is calculated at each time-step. A mean field approximation is used to track the average matrix solute level to be used in the next time-step.

(9) The time-step is incremented and the model is iterated until the end time is reached.

The resultant model is capable of predicting nucleation, growth, coarsening without artificial constraints, whether these processes occur concomitantly or sequentially. The model developed here uses a Runge-Kutta scheme to determine the time interval for each step to ensure model efficiency and numerical accuracy, and this is discussed in detail elsewhere [14].

The model relies on a number of input parameters, summarised in Table 1 . In particular, there are two parameters that have a large influence on the predictions but that are not known a priori. These are the interfacial energy of the SPPs, and the nucleation site density. Values for these parameters were taken directly from those used in previous work to model SPP formation in Zircaloy-2 [11], which themselves were derived from experiments on Zircaloy4 [2]. No further tuning of these values was undertaken since the purpose of the present model was not to provide a high level of quantitative accuracy (which would not be justified given the inherent approximations) but rather to demonstrate qualitatively how the SPPs should evolve under combined thermal and irradiation exposure. As demonstrated later, these parameters when used in the present model give a reasonable prediction of the particle size and number density after a simulated annealing treatment. 


\subsection{Summary of Model Assumptions}

Given the complexity of the processes controlling SPP evolution under irradiation and the uncertainty in some of the physical mechanisms it has been necessary to make a number of assumptions and approximations to produce a predictive model. One application of the model is to test the validity of these assumptions; i.e. if the predictions fail to match experiments this suggests an important mechanism has not been correctly captured. The model assumptions and approximations have been introduced already, but for clarity they are summarised here.

The model applies to the face-centred cubic $\mathrm{Zr}(\mathrm{Cr}, \mathrm{Fe})_{2}$ phase, which is the dominant SPP in Zircaloy-4. Other SPPs in other alloys systems (e.g. Zr- Nb alloys) behave differently. Only precipitation within grain interiors is considered; grain boundaries and the regions adjacent to them where solute is depleted are not considered. Isotropic diffusivity for solute is assumed, however experimental observations demonstrate the rate of SPP dissolution during irradiation can be directionally dependent $[9,6]$, which has been attributed to the known anisotropy of diffusion in the zirconium lattice [6]. Therefore, present model cannot predict the change in shape of the precipitates as they dissolve. Irradiation induced composition change and destabilization of SPPs is assumed to be controlled by ballistic scattering and is treated sequentially with particle dissolution; i.e. SPPs in the model will dissolve only once they becomes fully destabilised.

Reprecipitation of SPPs is not considered in the model. Reprecipitation is often observed to occur on heterogeneous nucleation sites such as grain boundaries [6]. This is not yet fully understood (for example, the reprecipitated phases are often observed to be different to the original SPPs) but could be incorporated in future in the KWN framework used in the present model. From a practical perspective, it is the initial dissolution of SPPs and commensurate change in matrix composition that will lead to a change in corrosion and other behaviour, and this is captured by the model.

\section{RESULTS}

\subsection{Initial particle size distribution}

The model was first applied to predict an initial condition that gives a mean SPP size and number density similar to that expected after commercial processing. In all simulations, the chemical composition assumed was assumed to 
be Zr-1.4Sn-0.2Fe-0.1Cr (wt\%), which corresponds to that of Zircaloy-4. In commercial processing, the particles evolve during a complex non-isothermal heat treatment, whereas the present model applies only to isothermal conditions. Therefore, an isothermal treatment was simulated that yields a predicted average particle size and number similar to that expected for the complex commercial treatment. A simulated treatment at $565^{\circ} \mathrm{C}$ for $2.5 \mathrm{~h}$ was found to give a reasonable match between parameters, as demonstrated later.

Figure 1 shows the predicted evolution of (a) precipitate volume fraction and matrix supersaturation, (b) precipitate number density and mean radius, and (c) final precipitate size distribution (PSD) for this annealing treatment. It can be seen that the nucleation stage of precipitation is complete very early during the simulated treatment, and after approximately $4 \mathrm{~s}$ nucleation is complete and most of the supersaturated solute has been precipitated. This rapid nucleation is consistent with previous simulations applied to a similar alloy (Zircaloy-2) [11]. Further evolution of the particles involves growth and coarsening. As discussed in detail elsewhere [16], and expected for systems with relatively high interfacial energy, there is a period where the shrinkage of small particles balances the growth of large particles and the mean radius and number density plateau. At times over approximately 6 minutes, the smallest particles dissolve completely and the number density falls, accompanied by an increase in mean radius. This is the classical coarsening regime, to which Lifshitz, Slyozov, Wagner (LSW) coarsening theory is usually applied [15]. It can be seen that at the end of the simulated anneal, the predicted particle number density has fallen to approximately one third of its maximum value. The PSD obtained after this treatment shows a log-normal distribution, which is expected after extensive particle coarsening. This PSD was used as the starting condition for all subsequent calculations.

The mean particles size and number density after this simulated treatment are $15.4 \mathrm{~nm}$ and $180 \mu \mathrm{m}^{-3}$ respectively. This can be compared to the expected results after a commercial (non-isothermal) process path of $20.0 \mathrm{~nm}$ and $100 \mu \mathrm{m}^{-3}$ [11]. Although the agreement is approximate and the PSD ob-

tained from an isothermal heat treatment is expected to be different from that obtained during non-isothermal processing, the exact form of the initial PSD is not critical. As shown later, regardless of initial PSD and mean particle parameters, a different PSD will become established relatively early during the simulated service exposure.

\subsection{Long Term Exposure: No Irradiation}

The model was then run for a lower temperature, long term exposure $(580 \mathrm{~K}$, 30 years), a temperature representative of that used in the experimental stud- 
ies for which the amorphization model was validated [8]. The irradiation model was first turned off; i.e. particles amorphization did not occur. The evolution of particle volume fraction, matrix solute level, mean particle radius, and number density is shown in Figure 2. Note that the matrix is slightly supersaturated at the start of this heat treatment because the simulation temperature is significantly lower than the initial annealing temperature. This is predicted to lead to a small amount of additional precipitate of SPP (a $2 \%$ change) but this is complete relatively early (after 5 days). It is unlikely that such a small volume fraction increase could be easily detected experimentally even if it occurs. This is accompanied by a small increase in the mean radius of particles.

After this time, the system is within the coarsening regime. As discussed above, at first the balance between dissolution and growth will lead to a plateau in the mean size at a constant number density. Eventually however, the smallest particles will fully dissolve and the number density starts to fall. The drop in number density is predicted to start at 11 days, but it is very gradual. However, at the end of the simulation (30 years) the particle number density is predicted to drop significantly (approximately a 50\% reduction). This is accompanied by an increase in mean particle radius of $25 \%$, with a final predicted mean radius of approximately $29 \mathrm{~nm}$. Therefore, even without irradiation, a significant change to the SPP size and number is predicted for very long term (30 year) thermal exposure.

\subsection{Long Term Exposure: With Irradiation}

The simulation was then run for the same thermal conditions already described ( $580 \mathrm{~K}, 30$ years) but with the irradiation model turned on and particle amorphization included. Note that 30 years is an order of magnitude longer than fuel is typically in service and was chosen to explore the effect of irradiation exposure out to times far longer than those used practically [12].

The displacement rate was taken as that for which the Motta model was validated, (i.e. $5 \times 10^{-7}$ dpa s$^{-1}$ [8] or approximately 15 dpa year $^{-1}$ ). The evolution of particle volume fraction, matrix solute level, mean particle radius and number density is shown in Figure 3, comparing the predictions with and without irradiation. The simulation results with and without irradiation are identical up until approximately 0.16 years (60 days).

Beyond this time, the predictions for the irradiated condition begin to deviate from those without irradiation due to amorphization and dissolution of SPPs. This leads to a fall in the number density which is far more rapid than that due to coarsening alone, combined with a sharp increase in the mean radius as the small particles become amorphized and dissolve first. Irradiation induced dis- 
solution leads to a reduction in volume fraction of SPPs and a complementary increase in the solute level in the matrix. The mean particle radius increases sharply under irradiation, as it is the largest particles that will survive the longest, before eventually becoming amorphized and dissolving. Eventually, full dissolution is predicted, at which point the mean particle radius abruptly falls to zero (this is due to the rapid dissolution of the particles in the largest occupied size class once they become fully amorphized). For the conditions used in this simulation, full dissolution of the SPPs is predicted to occur at 7.2 years. At this point, all of the initial solute is predicted to be dissolved back into the matrix, and there will be a high driving force for re-precipitation of new SPPs given suitable conditions.

The model was also run for a lower displacement rate of $3 \mathrm{dpa} \mathrm{year}^{-1}$, a value more typical of commercial reactor exposure [12]. The evolution of particle number density and mean radius are plotted for this case (compared with the no-irradiation and 15 dpa year $^{-1}$ case) in Figure 4. As would be expected, the lower displacement rate leads to a longer dissolution time. Even after the end of the simulation time (31.7 years) there remain a very small number of particles (0.0014 particles $\mu \mathrm{m}^{-3}$ ) predicted to remain undissolved, which are predicted to grow to a very large size ( $1 \mu \mathrm{m}$ radius). At a time more typical of end of fuel life (e.g. 3 years) it is predicted that approximately $10 \%$ of the SPPs remain (although it must be re-emphasised that the model is not expected to be quantitatively accurate),

Irradiation induced SPP dissolution will also have an influence on the PSD. The shape preserving log-normal distribution that is predicted during pure LSW coarsening is lost and replaced by an evolving PSD that changes over time. It is interesting to compare the PSD with and without irradiation at $t_{1 / 2}$ (Figure 5). $t_{1 / 2}$ is defined as the time taken to dissolve half of the original SPPs. Without irradiation, particle loss by coarsening alone gives $t_{1 / 2}=9.34 \times 10^{8} \mathrm{~s}$ (29.6 years) at $580 \mathrm{~K}$. With irradiation under the conditions used here, this time is predicted to be greatly reduced to $4.58 \times 10^{6} \mathrm{~s}(1.7$ months $)$. It can be seen that the PSD for the case without irradiation has the expected log-normal form that is predicted by LSW coarsening theory [15]. With irradiation, the predicted PSD is narrower and is also truncated at lower particle sizes. The truncation is due to the dissolution of small particles resulting from irradiation induced amorphization. The PSD is narrower because there has been less time for the largest particles to grow to the size they reach without irradiation (recall that when comparing these PSDs, that the total particle number density is the same, but the time to reach this number density is much lower when irradiation is included). Calculations for other times revealed that when irradiation is accounted for, the PSD never reaches a steady state shape, but evolves continuously with time. 


\subsection{Effect of Irradiation Displacement Rate}

The model was then applied to make predictions for a range of displacement rates $\left(1-20\right.$ dpa year $\left.^{-1}\right)$ at the same temperature used for previous calculations $(580 \mathrm{~K})$. The predicted evolution of SPP number density for 5 displacement rates in this range is plotted in Figure 6. As might be expected, a higher displacement rate leads to a more rapid decrease in the predicted number density of SPPs and a reduced time to full dissolution.

For comparison, $t_{1 / 2}$ is plotted as a function of displacement rate in Figure 7 . As displacement rate is increased, $t_{1 / 2}$ reduces, but the dependence is predicted to be non-linear, and is more sensitive to changes in displacement rate for lower rates. Although, given the approximations inherent in the model, the predictions are not expected to be quantitatively highly accurate, the very strong accelerating effect of irradiation in reducing the SPP number density predicted by the model is reasonable and consistent with experiment.

\subsection{Effect of Temperature}

The model was then run assuming a constant displacement rate $\left.\left(3 \mathrm{dpa} \mathrm{year}^{-1}\right)\right)$ for temperatures in the range $(473-673 \mathrm{~K}) 200-400^{\circ} \mathrm{C}$. The extremes of this temperature range are outside the limits that the amorphization model used in this work is likely to be valid $(520-580 \mathrm{~K})$. Nevertheless, applying the model over a wide temperature range allows an assessment of the effect of temperature, assuming the amorphization mechanism is not changed. By using a large temperature change, the effect of temperature is magnified to make clear the trends that will apply over the narrower temperature range for which the model will be valid.

The evolution of particle number density for temperature values evenly distributed in this range is shown in Figure 8. From such data, the time taken to reduce the original particle number density by $50 \%$ can be determined as a function of temperature. This is plotted in Figure 9.

The effect of temperature can be seen to be more complex than that of displacement rate; i.e. there is is not a monotonic increase (or decrease) in $t_{1 / 2}$ with temperature. The reason for this is a competition between two effects; amorphization and coarsening. This is explored in detail in the discussion. 


\section{DISCUSSION}

The aim of the model developed in this work was to provide an insight into the evolution of SPPs in zirconium alloys under irradiation by simplifying a complex problem to the most basic physical mechanisms operating, which are then allowed to interact with minimal artificial constraints.

The model predicts that even without irradiation, the SPP distribution in material entering service will not be stable during very long term thermal exposure. Since the processing temperatures are typically higher than the service temperature, there may be remnant solute supersaturation and this can lead to some nucleation of new SPPs and a small increase in volume fraction. Note that the thermal treatments used for commercial processing of zirconium alloys are not usually optimized with respect to SPP precipitation. Eventually, coarsening becomes the dominant mechanism leading to a reduction of particle number density and increase in mean radius. This is a slow process under thermal exposure alone (for example, it is predicted to take nearly 30 years to halve the particle number density due to coarsening at $580 \mathrm{~K}$ ).

When irradiation is introduced, the effects of classical coarsening are superimposed with the effect of irradiation induced amorphization and dissolution. This leads to a considerable increase in the complexity of the response of the SPP distribution to changes in exposure conditions (temperature and irradiation displacement rate). At a constant temperature, the predicted effect of increasing displacement rate is to strongly accelerate SPP dissolution. Even at modest displacement rates (e.g. 3 dpa year $^{-1}$ ) the time taken to dissolve half the SPPs is predicted to fall sharply (from approximately 30 years to 10 months at $580 \mathrm{~K}$ ). This is accompanied by a rapid increase in the size reached by the particles that remain undissolved at a given time.

As demonstrated, the effect of temperature on the SPP evolution under irradiation is more complex than that of displacement rate. Although it is certain that the model assumptions break down at the extremes of temperature to which it has been applied (e.g. at high temperature, diffusion driven recovery processes occur that are not considered in the model) the interaction between coarsening and irradiation induced SPP dissolution revealed by the model is physically sensible and will be important in determining the overall SPP evolution.

To understand the temperature effect on SPP evolution, it is instructive to plot the predicted change in particle number density for the same temperatures without irradiation, as shown in Figure 10. If this is compared to predictions when irradiation is active (Figure 8) it can be seen that as the temperature increases, the curves with and without irradiation effects converge. This can be 
seen more clearly by comparing the curves for 623 and $673 \mathrm{~K}\left(350\right.$ and $\left.400^{\circ} \mathrm{C}\right)$ only (Figure 11). At the highest temperature $\left(673 \mathrm{~K}, 400^{\circ} \mathrm{C}\right)$, the reduction in particle number density with and without irradiation is very similar and only begin to deviate at long times. This is because at high temperature, conventional coarsening dominates the loss of particles. As coarsening proceeds, the mean particle radius increases. Since larger particles take longer to become amorphous and dissolve, thermal coarsening serves to delay irradiation induced dissolution effects, so that they only play a significant role in reducing the particle number density at longer times. At the lower temperature of $623 \mathrm{~K}$ $\left(350^{\circ} \mathrm{C}\right)$, conventional coarsening is slower, and irradiation induced dissolution therefore becomes significant at shorter times. The curves with and without irradiation therefore diverge at shorter times, and the influence of irradiation is relatively greater.

There is thus a competition between thermal coarsening and irradiation induced amorphization and dissolution. Both processes will lead to a reduction in the SPP number density, but the relative importance of each will depend on the temperature. As the temperature increases, thermal coarsening will play a greater role in contributing to the dissolution of small SPPs. Thermal coarsening also influences the time for complete amorphization by increasing the average size of SPPs.

This interaction means that, depending on the temperature range, increasing temperature is predicted to either increase or decrease the time to dissolve half of the SPPs (i.e. $t_{1 / 2}$ ). Over the limited temperature range for which the model assumptions are likely to be valid, this interaction is predicted to lead to a weak temperature dependence on the time for SPP dissolution (weaker than would be expected from coarsening alone without irradiation). A key implication of these predictions, which is counterintuitive based on the conventional understanding of non-irradiated alloys, is that coarsening may delay full dissolution of SPPs by producing particles which are larger, and therefore more resistant to irradiation damage and thus dissolution.

In summary, although the approximations inherent in the model mean its quantitative predictions are not expected to be highly accurate, the interaction of coarsening and irradiation that has been identified is likely to be important in understanding how service conditions influence the susceptibility of SPPs to irradiation induced dissolution. Furthermore, the model suggest several key areas where further experiments are needed to provide the understanding and data required to improve its fidelity. 


\section{CONCLUSIONS}

A coupled model has been developed to predict the evolution of second phase precipitates (SPPs) in alloys subject to thermal and irradiation exposure. The model combines a classical size class model for precipitate nucleation, growth, and coarsening with a simple model for irradiation induced precipitate amorphization and dissolution. The model has been applied to the case of SPP evolution in Zircaloy-4. The following conclusions may be drawn from this work:

(1) It is predicted that at typical service temperatures, the equilibrium volume fraction of SPPs will relatively quickly become established ( 5 days at $580 \mathrm{~K})$, after which further changes in the absence of irradiation will be dominated by the coarsening process.

(2) Even without irradiation, coarsening will lead to SPP dissolution, and a slow reduction in number density, which is predicted to start at around 11 days (at $580 \mathrm{~K}$ ) and lead to a halving in SPP number after 30 year exposure.

(3) Irradiation is predicted to strongly accelerate SPP dissolution, even at modest displacment rates $\left(<3\right.$ dpa year $\left.{ }^{-1}\right)$. The effect is predicted to be non-linear with increasing displacement rate, with greater sensitivity at low values.

(4) The model reveals a complex interaction between temperature and irradiation induced displacement rate in determining SPP evolution. As temperature increases, conventional coarsening becomes more important than irradiation induced dissolution in determining the overall SPP number density. For typical commercial reactor conditions, irradiation induced dissolution is dominant.

(5) Thermal coarsening may increase resistance to irradiation induced amorphization and hence delay the time to fully dissolve the SPPs by increasing the mean SPP size.

(6) The model has highlighted several key areas where greater understanding is needed regarding the interaction of precipitation and amorphization. In particular, the reprecipitation processes that are expected once the supersaturation exceeds a certain level dur to SPP dissolution are not yet understood in sufficient detail to be included in the model.

\section{References}

[1] F. Garzarolli, H. Stehle, in: Proc. Int. Symp. on Improvements in Water Reactor Fuel Technology and Utilization, IAEA-SM-288/24, 1986, pp. 388-407.

[2] J. P. Gros, J. F. Wadier, J. Nucl. Mater. 172 (1990) 85-96. 
[3] M. Griffiths, R. W. Gilbert, G. J. C. Carpenter, J. Nucl. Mater. 150 (1987) $53-66$.

[4] M. Griffiths, J. Nucl. Mater. 159 (1988) 190-218.

[5] E. M. Francis, A. Harte, P. Frankel, S. J. Haigh, D. Jädernäs, J. Romero, L. Hallstadius, M. Preuss, J. Nucl. Mater. 454 (2014) 387-397.

[6] M. Griffiths, J. Nucl. Mater. 170 (1990) 294-300.

[7] D. Pêcheur, F. Lefebvre, A. T. Motta, C. Lemaignan, D. Charquet, J. Nucl. Mater. 205 (1993) 445-451.

[8] A. T. Motta, C. Lemaignan, J. Nucl. Mater. 195 (1992) 277-285.

[9] W. J. S. Yang, J. Nucl. Mater. 158 (1988) 71-80.

[10] R. Tendler, J. P. Abriata, J. Nucl. Mater. 150 (1987) 251-258.

[11] A. R. Massih, L. O. Jernkvist, Computational Materials Science 39 (2007) 349-358.

[12] W. Hoffelner, Material for Nuclear Plants, Springer London, 2013, Ch. Irradiation Damage, pp. 255-307.

[13] R. Kampmann, R. Wagner, Materials Science and Technology, vol. 5, VCH Weinheim, Germany, 1991.

[14] J. D. Robson, M. J. Jones, P. B. Prangnell, Acta Materialia 51 (2003) 1453.

[15] J. W. Christian, Theory of Transformations in Metals and Alloys, Part 1, Pergamon Press, Oxford, 1975.

[16] J. D. Robson, Acta Mater. 52 (2004) 4669-4676. 

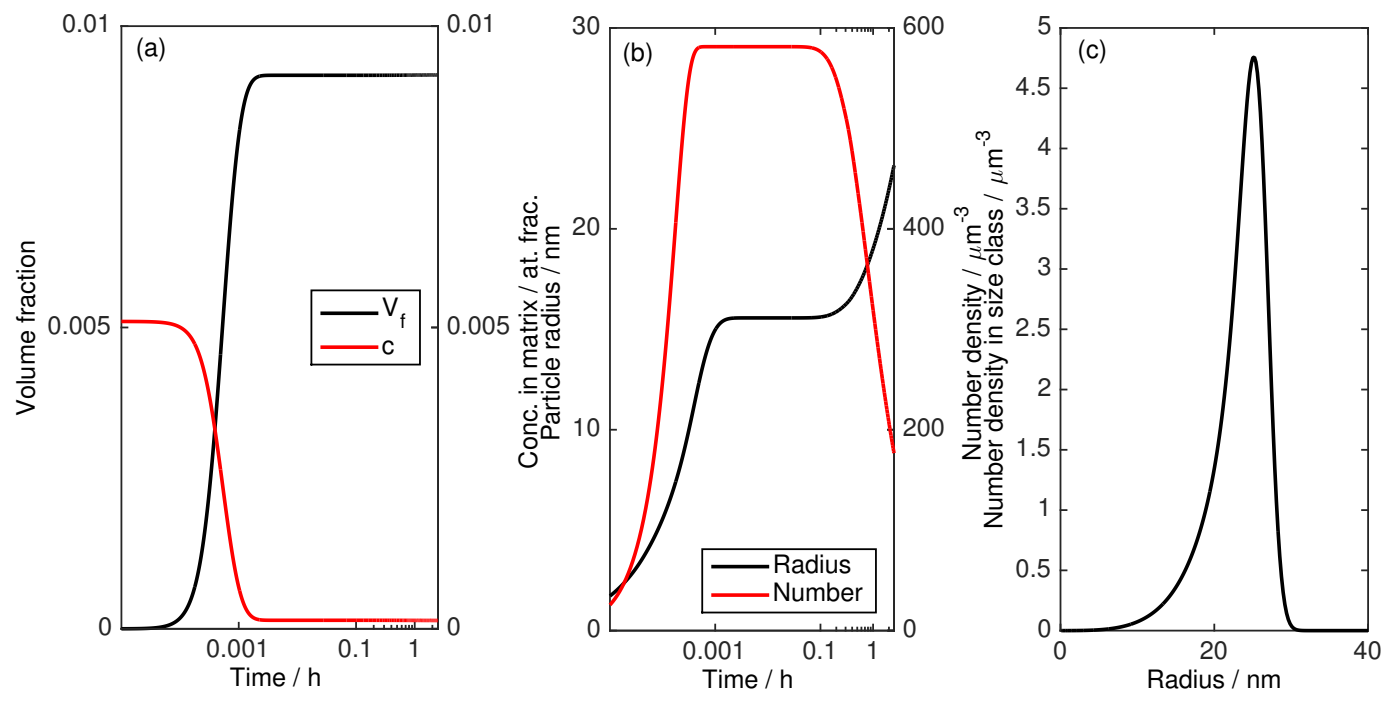

Fig. 1. Predicted evolution of precipitates during simulated annealing treatment (a) precipitate volume fraction and solute concentration in matrix, (b) mean precipitate radius and number density, (c) particle size distribution at the end of annealing.

Fig. 2. Predicted evolution of precipitates during long term (30 year) thermal exposure at $580 \mathrm{~K}\left(307^{\circ} \mathrm{C}\right)$ without irradiation (a) precipitate volume fraction and solute concentration in matrix, (b) mean precipitate radius, (c) precipitate number density. 
Fig. 3. Prediction evolution of (a) SPP volume fraction and matrix solute level, (b) SPP mean radius and number density at $580 \mathrm{~K}\left(307^{\circ} \mathrm{C}\right)$ without and with irradiation (displacement rate 15 dpa year ${ }^{-1}$ ).
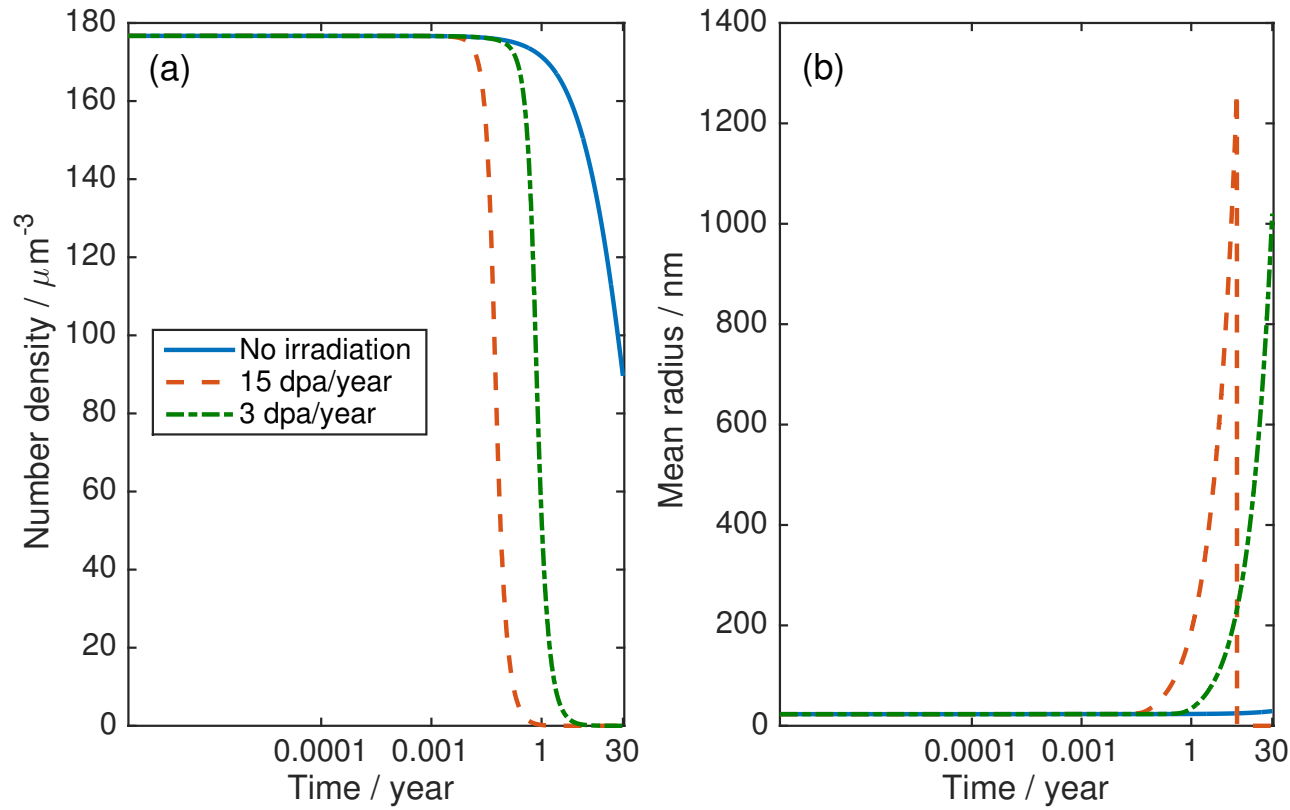

Fig. 4. Predicted evolution of (a) SPP number density (b) mean particle radius at $580 \mathrm{~K}\left(307^{\circ} \mathrm{C}\right)$ for the cases of no irradiation, irradiation at $3 \mathrm{dpa}_{\text {year }}{ }^{-1}$, irradiation at 15 dpa year ${ }^{-1}$. 


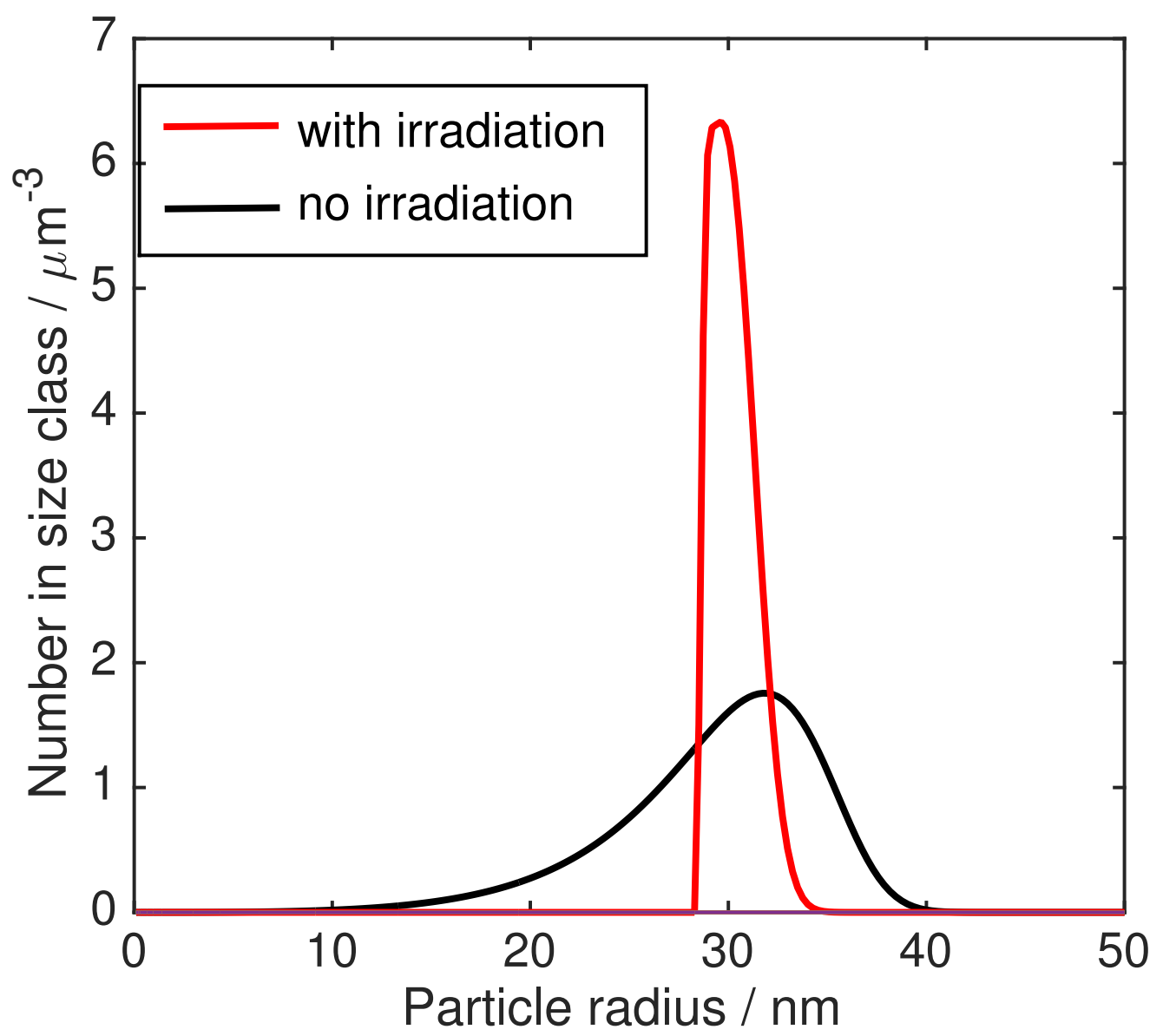

Fig. 5. Predicted particle size distribution (PSD) at the critical time required to dissolve half the original SPPs $\left(t_{1 / 2}\right)$ without and with irradiation at 15 dpa year $^{-1}$. 


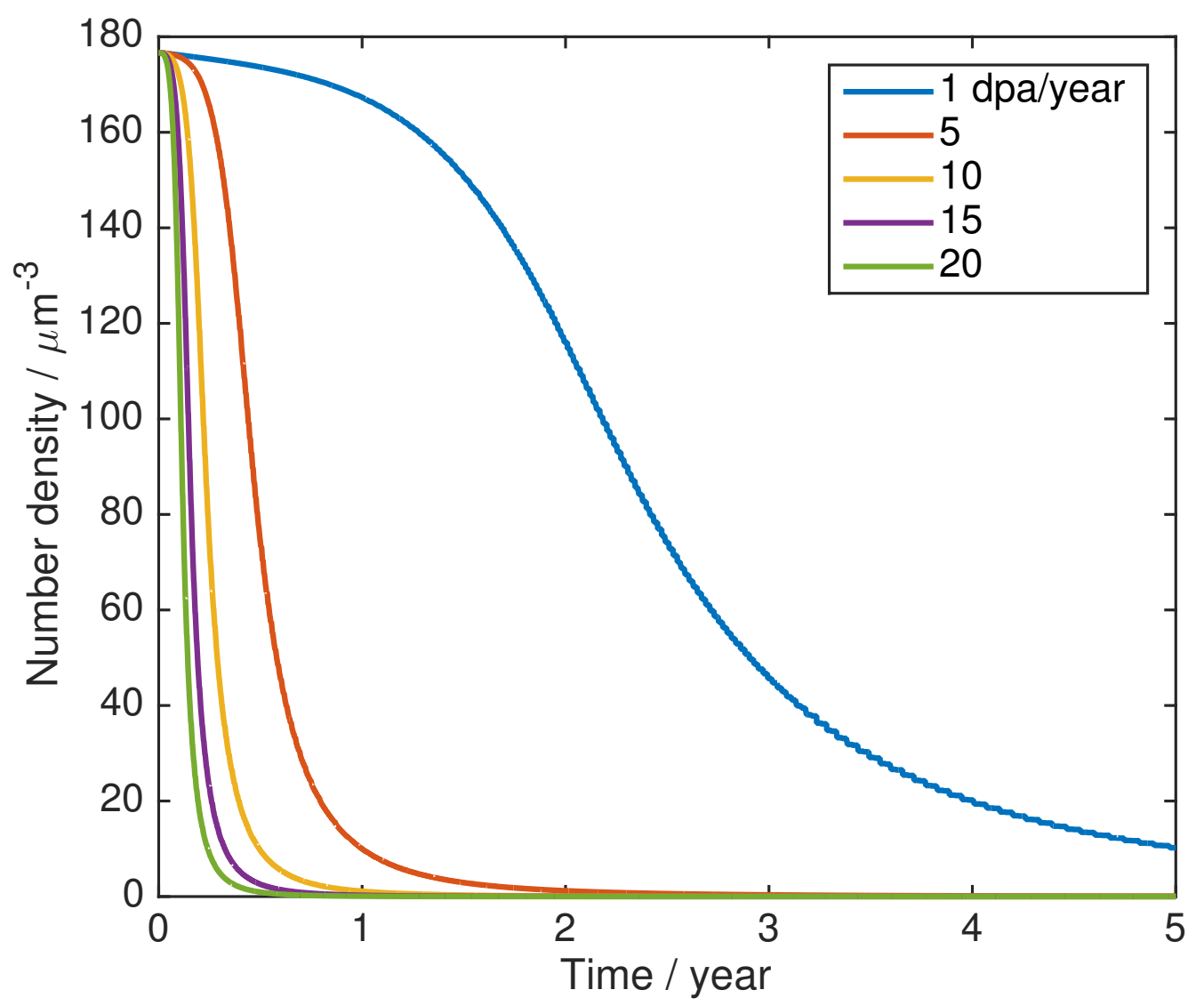

Fig. 6. Predicted evolution of SPP number density at $580 \mathrm{~K}\left(307^{\circ} \mathrm{C}\right)$ for five displacement rates (1-20 dpa year $\left.{ }^{-1}\right)$. 


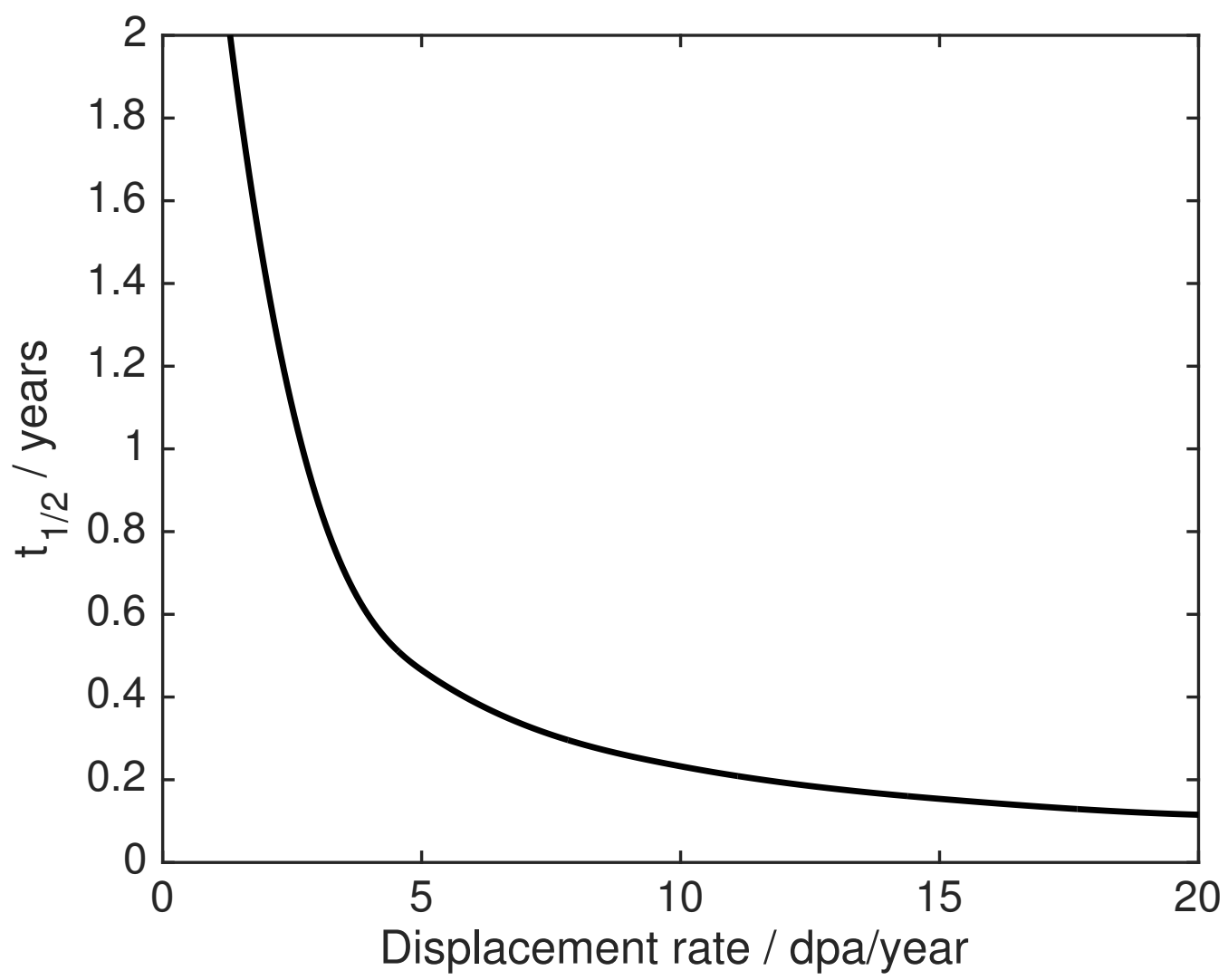

Fig. 7. Predicted time to dissolve half the $\operatorname{SPPs}\left(t_{\frac{1}{2}}\right)$ as a function of displacement rate. 


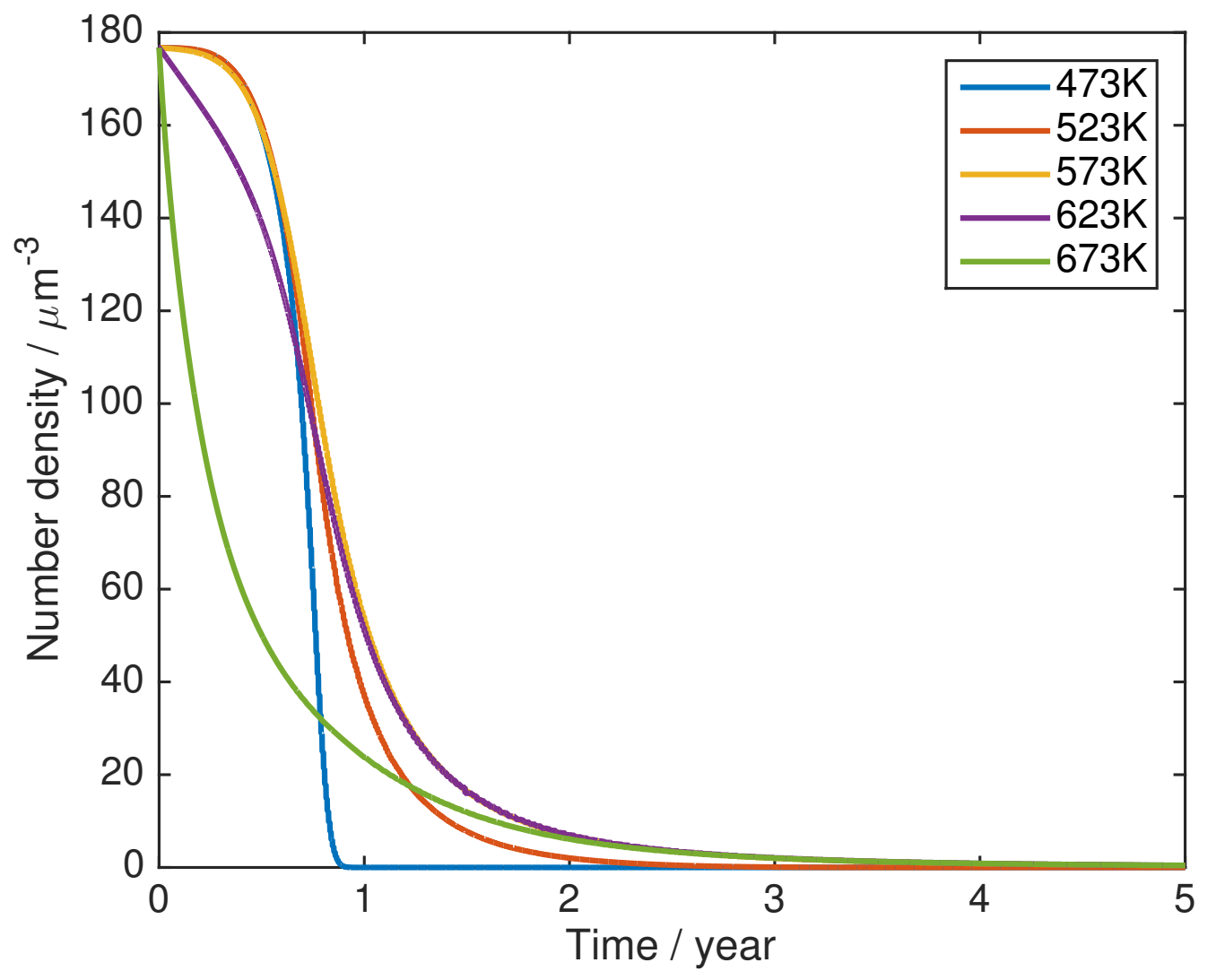

Fig. 8. Predicted evolution of SPP number density for a displacement rate of 3 dpa year $^{-1}$ and temperatures in the range $473-673 \mathrm{~K}\left(200-400^{\circ} \mathrm{C}\right)$. 


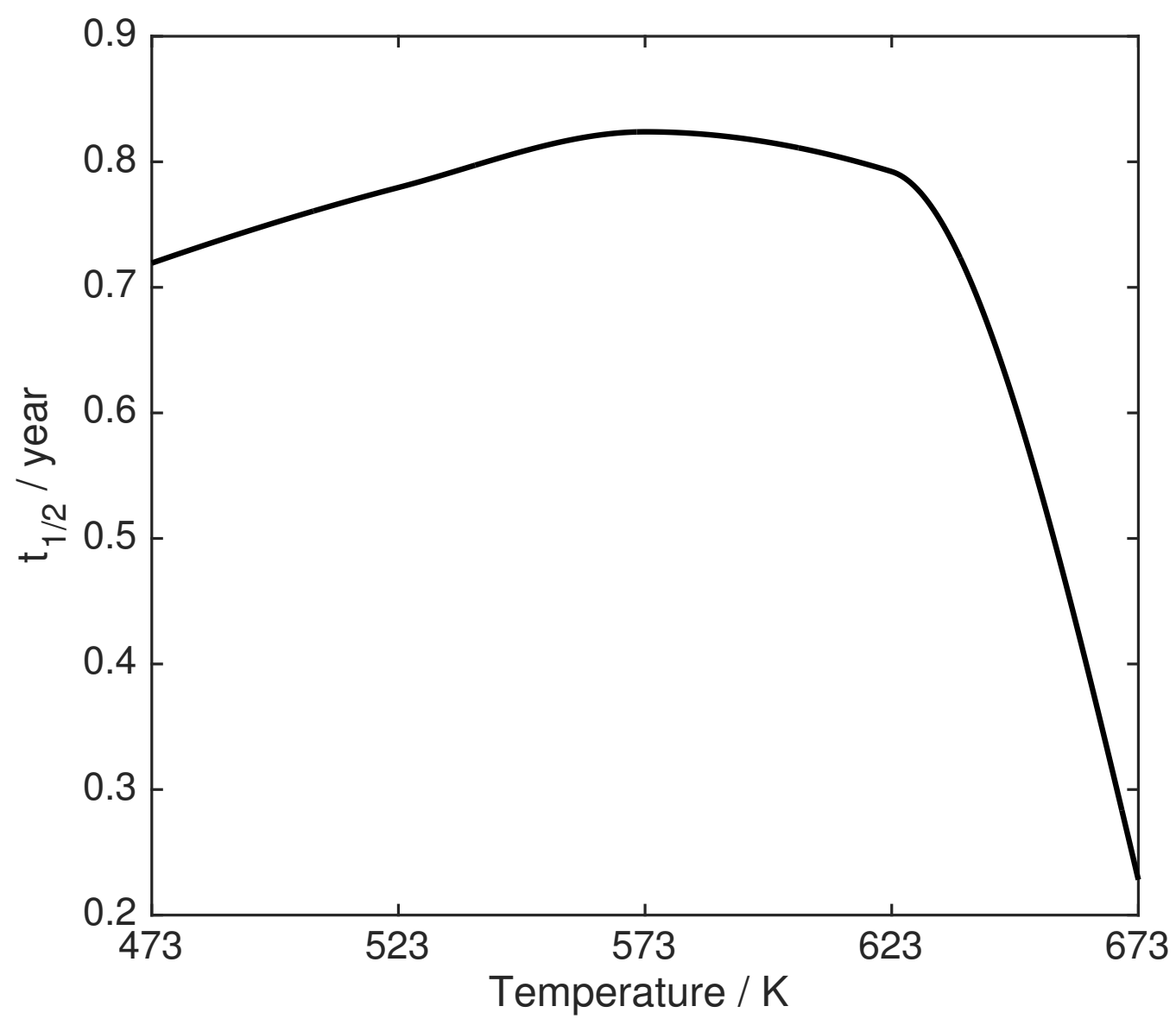

Fig. 9. Predicted effect of temperature on the time to dissolve half of the SPPs for a displacement rate of 3 dpa year ${ }^{-1}$. 


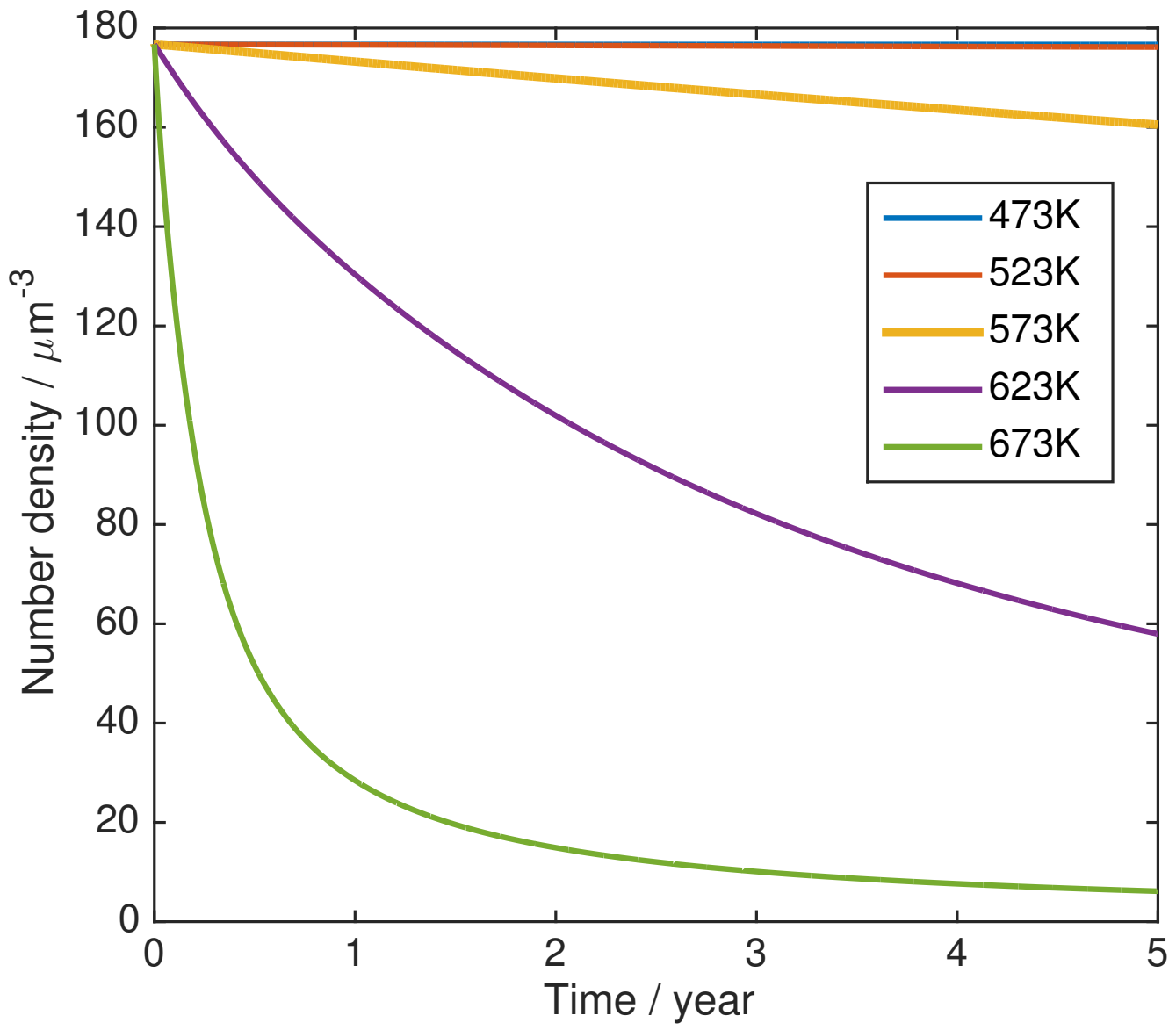

Fig. 10. Predicted evolution of SPP number density without irradiation for temperatures in the range $473-673 \mathrm{~K}\left(200-400^{\circ} \mathrm{C}\right)$. 


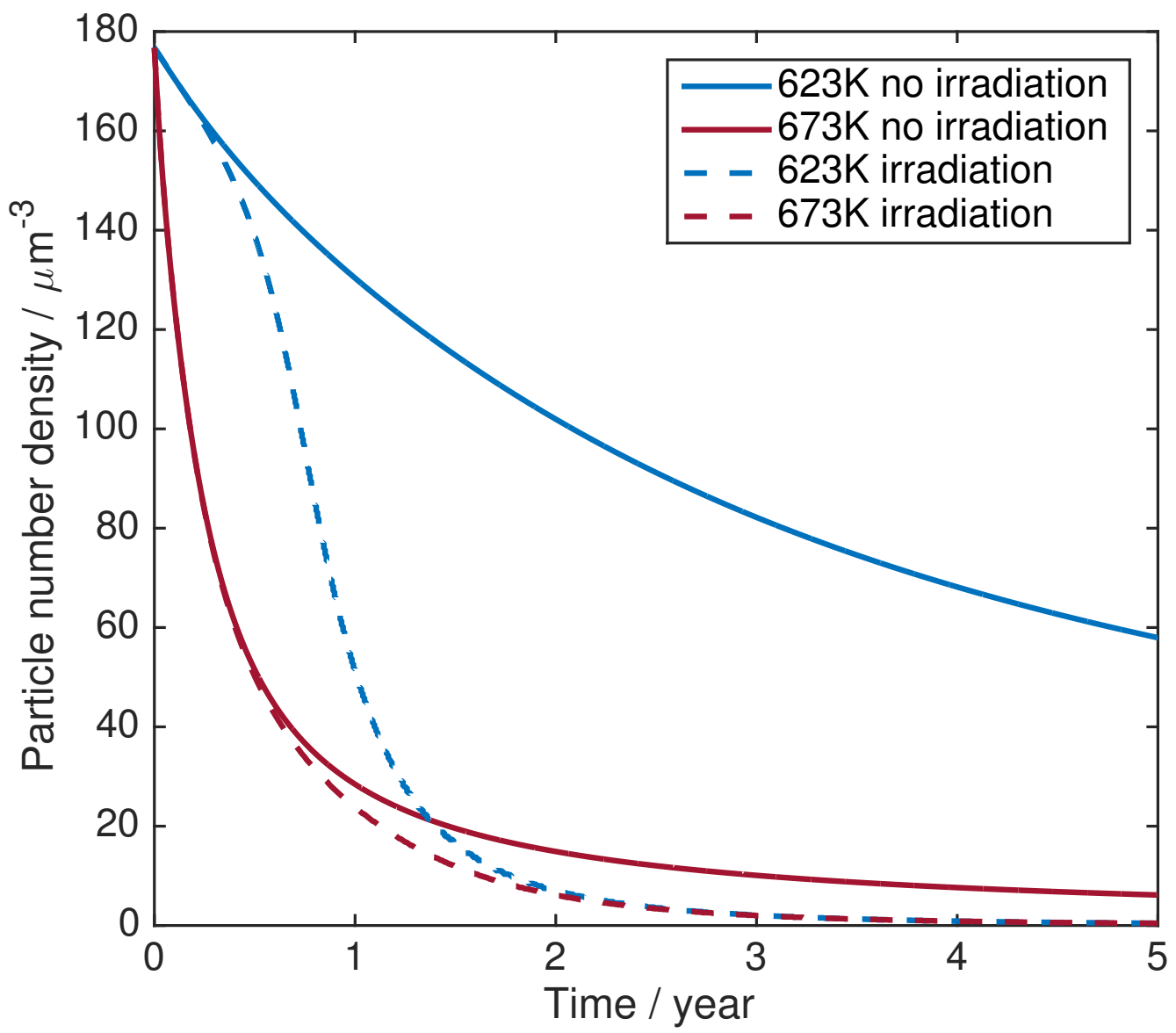

Fig. 11. Predicted evolution of SPP number density without and with irradiation (at 3 dpa year $\left.^{-1}\right)$ for 623 and $673 \mathrm{~K}\left(350\right.$ and $\left.400^{\circ} \mathrm{C}\right)$. 